velle géographie économique, nouvelle économie sociale... nouvelle cuisine (dans ce dernier cas, c'est, hélas, une réalité). Reprenant leur cercle d'or, les auteurs y rattachent cette fois pas moins de douze composantes pour décrire ce qu'est un territoire solidaire. On tourne une roue qui va de la richesse à la gouvernance, en passant, entre autres, par les projets et la cohésion sociale. Des travaux en cours sur les Pactes ruraux, élément-clé de la politique québécoise de la ruralité, permettront peut-être d'identifier de tels territoires dits solidaires.

Certains de mes collègues de 1'Université du Québec à Montréal et de l'Université du Québec en Outaouais, très portés sur l'économie sociale, apprécieront les nombreuses allusions faites à cette (nouvelle ?) discipline à laquelle les auteurs accolent l'économie solidaire vue comme l'ensemble des initiatives qui se développent autour de valeurs de réciprocité, de citoyenneté et de collectivité. Ce qui rappellera aux gens de ma génération ce que l'on désignait à une certaine époque sous l'appellation « économie communautaire ».

La section «Comprendre la dynamique des territoires » à l'intérieur du troisième et dernier chapitre portant sur les territoires créatifs contient un grande nombre d'illustrations présentées sous la forme de radioscopies. Ainsi, à titre d'exemple, à Dieppe, en relation avec la logique des réseaux, on signale pour le port de pêche 1747 tonnes de coquilles Saint-Jacques, 2700 tonnes de poisson, 53 bateaux, 256 marins (sic) et un nombre indéterminé de mareyeurs... On comprendra que le lecteur est ici invité à tourner les pages à la recherche de l'essentiel. Et il trouvera, entre autres, dans une section sur la nécessité de penser les territoires à travers les logiques d'acteurs, un intéressant quadrant mettant en relation territoire projet - territoire créatif - territoire soumis - territoire réceptacle. Le tout se termine de façon brusque, sans la moindre conclusion, par un schéma voulant montrer que l'économie cognitive favorise l'échange des savoir, alors que l'économie sociale et solidaire rappelle que la société du vivre ensemble met l'homme au centre du sujet. Au moment de la «montée en puissance » de l'économie libérale (milieu du $\mathrm{XIX}^{\mathrm{e}}$ siècle), Thomas Carlyle disait que la théorie qui lui servait de base n'était rien d'autre qu'une science funeste (dismal science). À la lecture de ce livre, il admettrait que la science économique peut être humaine.

\section{Christian Schmitt (sous la direction de) \\ Université et entrepreneuriat : une relation en quête de sens Paris, L'Harmattan 2005, 315 pages}

Voici un volume qui traite de l'université comme une organisation susceptible d'intervenir pour le mieux être de son territoire via l'entrepreneuriat. De « quoi » s'agit-il ? Et « comment» parvenir à établir une relation entre l'université et la création d'entreprise ? Tel est le questionnement sous-jacent de cet ouvrage auquel pas moins de dix collègues ont été appelés à collaborer. Le responsable de l'ouvrage, Christian Schmitt, docteur en gestion, est maitre de conférences à l'ENSAIA (École nationale supérieure d'agronomie et des industries alimentaires) à l'INPL (Institut national polytechnique de Lorraine) et chercheur au GREFIGE (Groupe de recherche en économie financière et gestion des entreprises) (la France et ses sigles...) à la ville des bergamotes (bonbons qui font la renommée de Nancy). C'est sans surprise que l'on trouve dans sa note biographique une allusion à ses travaux sur la relation entre l'université et l'entrepreneuriat. Il est aussi mentionné qu'il fut professeur invité à l'INRPME (Institut de recherche sur les PME) de l'UQTR (Université du Québec à Trois-Rivières), avec les conséquences que je mentionne plus loin. Cet ouvrage, tel que bien précisé en introduction, se veut être une vitrine des pratiques dans les domaines de la formation, de la recherche et de la valorisation de l'entrepreneuriat au sein de l'université. Pour ce faire, on offre au lecteur des expériences menées en Iran, au Vietnam, au Maroc, en Turquie, au Mexique, en Belgique et finalement... au Québec. Pas en France ? Enfin, nous pouvons toujours croire que la mère-patrie fait partie des réflexions, de façon implicite, que nous fournit notre collègue lorrain dans un intéressant chapitre qu'il co-signe avec deux collaborateurs.

Il serait fastidieux de résumer la gamme variée des initiatives mises en évidence ici et là à la faveur des différents chapitres. Elles intéressent avant tout les acteurs directement impliqués dans une telle démarche pour voir les similitudes et surtout, tel qu'indiqué, toujours en introduction, les distinctions. Le lecteur intéressé trouvera à n'en pas douter des leçons à tirer de nature à voir une mise en application, toute chose étant égale par ailleurs, comme le veut l'adage si cher aux économistes (citeris parebus). Robert Paturel, 
professeur de management stratégique et d'entrepreneuriat à l'Université du Sud de Toulon-Var, est responsable des chapitres traitant de l'Iran, du Vietnam et de la Turquie, mais rien n'indique qu'il y soit effectivement allé. Souhaitons-le lui. À tout effet, il a su très bien s'acquitter de sa tâche.

Le chapitre qui a surtout retenu mon attention est le sixième intitulé "Université et entrepreneuriat : vers le développement d'une relation paradoxale ? ». Pour tenter de répondre à la question, Christian Schmitt a bénéficié du concours de Sandrine Berger-Douce, maître de conférences à l'IAE (Institut d'administration des entreprises) de Valenciennes, et de Mohamed Bayad, professeur des universités et directeur d'un groupe de recherche de l'Université Nancy 2. Leur questionnement porte plus particulièrement sur la place des incubateurs universitaires. Ceci, après avoir cherché à mieux comprendre la construction à travers le temps de la relation entre l'université et l'entrepreneuriat. Le tout commence donc par une présentation en quatre phases : de l'absence totale de préoccupation envers l'entrepreneuriat à celle de l'intégration. Cette dernière comprend trois missions: formation, recherche et valorisation. C'est ce qui permet l'implantation d'un incubateur. Sur la base de leur propre expérience et sur un rapport d'Ernst \& Young (2003) (ce rapport doit être drôlement connu car on ne le retrouve pas en bibliographie...) il apparaît que le rôle de l'université à travers ses trois missions se perçoit mal et que la venue des incubateurs a pour effet de faire émerger de nouveaux types de relations entre l'université et l'entrepreneuriat. Une lecture attentive permet sûrement de comprendre les subtilités de la situation paradoxale que les auteurs mettent en évidence. C'est sur la base de cette réflexion qu'ils en arrivent à proposer un dispositif plus complet de l'entrepreneuriat au sein de l'université, évitant ainsi de se limiter à une relation linéaire allant de la détection à la création d'entreprise.

Le tour d'horizon de la planète se termine par une contribution de mon collègue Pierre-André Julien à qui Christian Schmitt ne pouvait se permettre de ne pas transmettre une invitation à collaborer à l'ouvrage puisqu'il faisait un stage à l'INRPME. On passe alors à un tout autre sujet : le partenariat entre l'université et une très grande entreprise, en l'occurrence Bombardier. Ce qui ne manque pas, par ailleurs, d'intérêt, là n'est pas le problème. J'ai déjà fait part de l'érudition de mon collègue dans ces pages (vol 14, $\mathrm{n}^{\circ} 2$ ).
Fidèle à sa réputation, il offre au lecteur, dans un chapitre de 20 pages, pas moins de sept (oui! 7) pages de références bibliographies qu'il (je le dis à nouveau) a sûrement lues. Comment ne pas trouver intéressantes des allusions à l'alchimie de Gerber ( $\mathrm{X}^{\mathrm{e}}$ siècle) ou encore aux non moins connus (!) Kaswuny et AbdallaTif qui, au XIII ${ }^{\mathrm{e}}$ siècle, facilitèrent le commerce avec l'Afrique noire et la Chine ? Mais comme le disait mon oncle Télesphore, "ça nous éloigne du sujet en titi ». Car, s'il y a bien un incubateur (l'objet du volume) sur le campus de l'UQTR, à quelque cent mètres de l'INRPME, il n'en n'est pas question dans ce chapitre. Avoir eu la chance de croiser Christian Schmitt lors de son séjour dans la ville de Laviolette, je lui aurais fortement conseillé de faire appel à la contribution de nos amis Yvon Gasse et/ou LouisJacques Filion, ou encore à Louis Dussault qui, eux, à n'en pas douter, s'investissent depuis des lustres dans ce qui fait l'objet de ce livre. Et qui sait ? Je lui aurais peut-être proposé mon humble apport à partir de mon expérience au Brésil. Croyez-vous que je sois jaaaaaaaloux? Paaaaass du tout! Pas du tout! (sur un air connu). Je n'aime pas les ouvrages collectifs, même si j'ai collaboré à une vingtaine (parfois à mon corps défendant) et, probablement, à cause du fait que j'ai été appelé à en recenser une cinquantaine, et c'est pas fini...

Pour en revenir à nos pâturages, Bayad et Schmitt concluent en évoquant la diversité des modèles observés. De ce périple autour du globe, ils dégagent quelques enseignements utiles. Ainsi, ils signalent qu'à chaque élément renvoyant aux fameux trois «I » (Intégration, Initialisation, Institutionnalisation) correspond un modèle de l'entrepreneuriat spécifique sur lequel l'université s'est appuyée. Sur la base de différents travaux, ils en arrivent à dégager trois modèles : de la réalisation; de la valorisation; de la conception. Et, enfin, comment ne pas être d'accord avec eux lorsqu'ils affirment être maintenant en mesure de parler d'un entrepreneuriat universitaire ? Cependant, quand ils disent que cette forme d'entrepreneuriat est largement absente des recherches dans le domaine, je suis tenté de leur dire ceci : à l'occasion de leur prochain voyage (pour voir les baleines, ce qui les changera de petits poissons des chenaux), ils devraient faire un arrêt à l'Université Laval et à l'École des Hautes études commerciales de Montréal. En attendant, je recommande la lecture de cet ouvrage à ceux qui, par leur expérience, trouvent déjà un certain sens dans la relation entre l'université et l'entrepreneuriat. 